\title{
THE INTRINSIC NATURE OF EMERGENCE-WITH ILLUSTRATIONS
}

\author{
Vincent Vesterby
}

\section{INTRODUCTION-THE PROBLEM}

Over a hundred years ago, George Henry Lewes (Lewes, 1891) introduced anthropomorphism into the emergence literature. "Thus, although each effect is the resultant of its components, the product of its factors, we cannot always trace the steps of the process, so as to see in the product the mode of operation of each factor. In this latter case, I propose to call the effect an emergent."

Material-reality consists of patterns-of-material-organization, from quarks and elementary particles to galactic clusters and the universe. The general systems factor, emergence, as discussed by Mill (Mill, 1843), and as described by Lewes in the first part of the above quote, is the coming into existence of new pattern of materialorganization - the general process by which all newly existing material pattern originates. Whether or not humans can trace the steps of the process of coming into existence is not relevant to what emergence is, or to the roles it plays throughout the universe.

Identifying emergence with a factor extrinsic to emergence itself (human knowledge or ignorance of the steps of the process), has resulted in many attempts to define emergence with factors that are not relevant to its intrinsic nature-(a) surprise, (b) novelty, (c) nonreducibility, (d) indeterminism, (e) unpredictability, (f) that there are qualities of the whole that do not occur as qualities of any of the parts, and so on. The consequence is confusion concerning the nature of emergence and its roles in creating the organization of material-reality.

Another, closely related factor, anthropocentrism, has also been contributing to the confusion concerning emergence. Anthropocentrism occurs when interpreting reality primarily through human experiences, values, and goals interferes with the accurate analysis and understanding of the intrinsic nature of reality (of that which exists). This problem occurs in the emergence literature where authors spend considerably more time discussing concepts concerning emergence than they do discussing emergence itself. Concepts are epistemological factors, and occur in the universe only where there are minds capable of having concepts - which all together constitute an extremely small part of reality. Emergence, the reality-referent of the concept of emergence, is omnipresent in multiple forms throughout material-reality. To focus primarily on concepts rather than their reality-referents is anthropocentric, and results in unrealistic opinions and conclusions. This problem is compounded by the common failure to distinguish in a sentence or a paragraph whether a word, phrase, clause, or sentence refers to the concept of emergence or to the reality referent of that concept.

This paper does not discuss the concept of emergence. Concepts, here, are used to direct the mind to the reality referent, to emergence itself. 


\section{Intrinsic Nature of Emergence-With Illustrations}

\section{GENERAL CONTEXT}

All material-reality that we experience is foundationally composed of elementary particles. These particles - electrons, protons, neutrons, photons, and so on-are organized into seemingly endless patterns-of-organization of material structure and process. These patterns range in size and complexity from protons and the simple hierarchies of atoms and molecules, to the awesome complexities of planets, ecosystems, brains, and civilizations, and further, to the immensities of galactic clusters and the infinite universe (Figure 1). The difference between the smaller or the simpler patterns and the larger or the more complex patterns is the quantity of elementary particles and the patterns-of-organization of those particles. The difference between a carbon atom and a rainforest, beyond the number of elementary particles involved in each, is the organization of those particles (Figure 2). Beyond quantity, all the factors that distinguish a molecule of deoxyribonucleic acid from our current global civilization are the specific patterns-of-organization that play roles in each of them and thereby make each of them what they are.

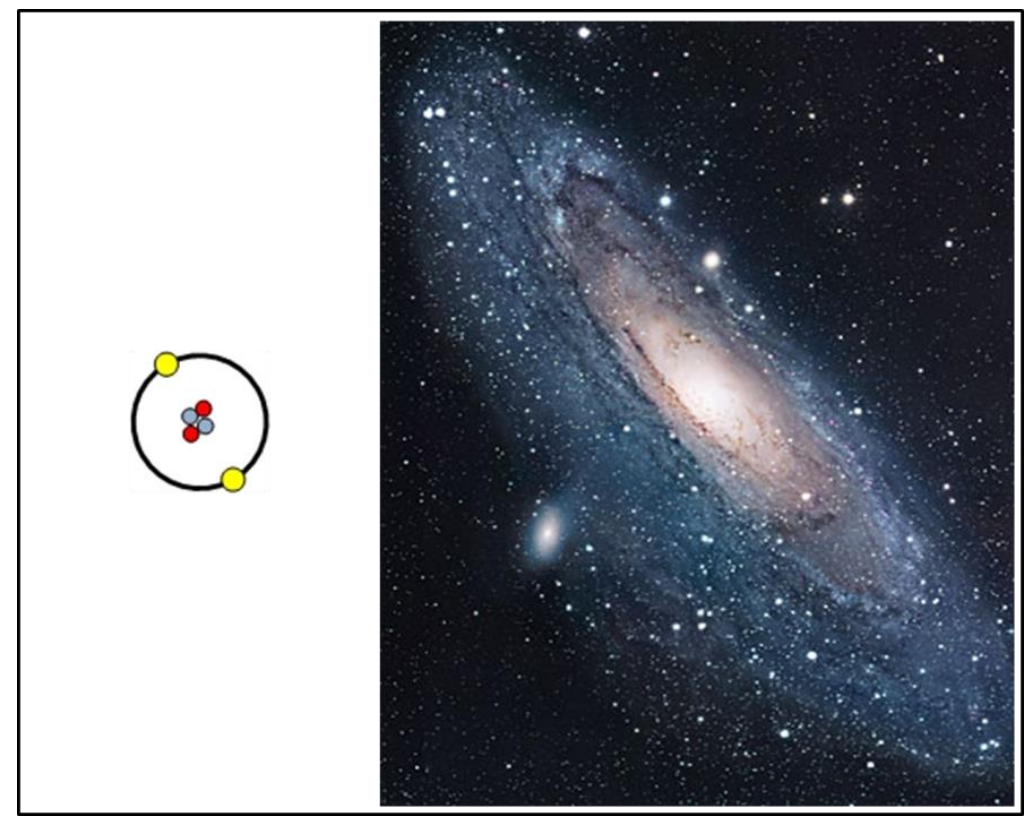

Figure 1. The Context of Emergence. Patterns-of-organization-for example atoms and galaxies. (2002 R. Gendler, Photo by R. Gendler) 


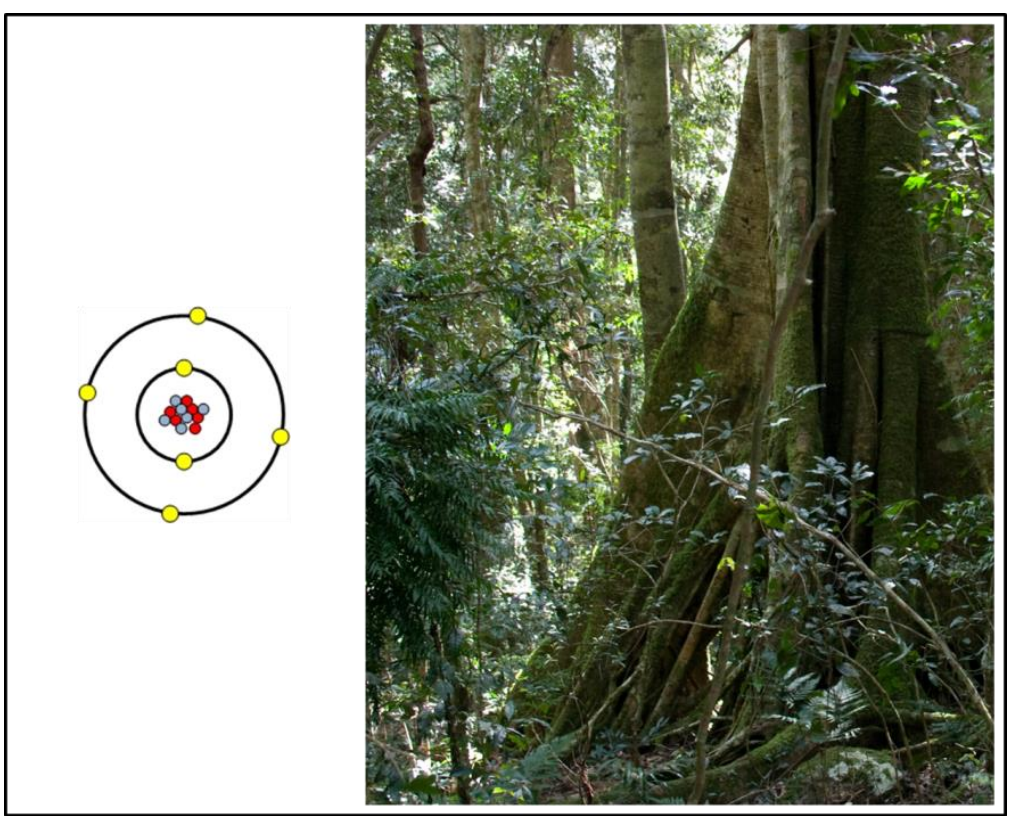

Figure 2. The Context of Emergence. A carbon atom and a rainforest differ in number of elementary particles and in the respective patterns-of-organization of those particles.

General systems is the field that studies these patterns-of-organization in broad scope, as they occur throughout the breadth and depth of material-reality. General systems studies them further, in detail — what they are intrinsically, and the manner in which they determine the nature of the different situations and systems in which they occur (Bertalanffy, 1968).

\section{The General Systems Context for Understanding Emergence}

A factor is something that exists and plays a role in the situation or system in which it exists. A situation is a combination of interrelated factors. A pattern-of-organization that exists in a situation and plays a role there is a factor of that situation. A general-factor is something that exists and plays a role in the intrinsic nature of two to many different situations. General systems is particularly interested in patterns-of-organization that are general-factors because they provide great breadth and depth of understanding.

Reality is that which exists. There is but one reality - all that exists. Reality develops, that is, everything that exists takes part in one way or another in a universally omnipresent transition, a sequential-difference from one time, place, part, pattern, level, condition, or situation to another involving some form of enhancement (Vesterby, 2008b). Development occurs in many different forms, the most obvious of which are time, motion, material transformation, and the hierarchic organization of matter from quarks to the pattern-of-organization of galactic clusters throughout the universe. All the different forms of development, and all the individual cases of each form throughout the infinite universe, together constitute the general-development-of reality. 


\section{Intrinsic Nature of Emergence-With Illustrations}

Each form of development is based on some form of sequential-difference. The various patterns-of-organization of material structure and process can occur in these developmental sequences wherever the intrinsic qualities of the developing situations can support or take on the form of the patterns-of-organization. It is typical of specific patterns-of-organization to occur at specific developmental stages or specific hierarchic levels in a developmental sequence.

A development-of-origin is a stage or a level in some form of development where a factor (such as a specific pattern-of-organization) comes into existence in that sequential development. The foundational-development-of-origin of a factor is the stage in the general-development-of-reality where that factor first comes into existence.

Situation-development is the combined concurrent developments of all the components of a situation together with the combined concurrent developments of all the interrelations between those components.

Existential-pathway-development is the continuously ongoing sequentially connected development of a situation. This is the continuous developmental pathway taken by situation development.

With factor-development, general-factors tend to occur in simpler forms in situations where few factors are playing roles, and to occur in more complex forms in more complex situations where greater numbers of other factors are playing roles. While general-factors have their intrinsic self-identity throughout their development, their forms and roles can be modified by what it is that constitutes their material basis at various stages and levels, and by the nature of the situations in which they occur. (Vesterby, 2008a)

Deep-structure consists of the underlying patterns-of-organization of structure and process of lower levels of the hierarchic organization of material-reality that result in the existence and organization of structure and process of upper levels. With material reality, deep-structure extends from the level of elementary particles to the level developmentally just below the top level of an object, system, or situation.

\section{THE INTRINSIC NATURE OF EMERGENCE}

Emergence is a general-factor, a pattern-of-organization that plays a universal role in process, in materially based change. Emergence is the coming into existence of new pattern-of-material-organization as a consequence of motion. It is the general process by which all newly existing material-pattern originates.

In the general-development-of-reality from simplicity to complexity, the foundationaldevelopment-of-origin of emergence occurs when the motion of one unit of matter changes the distance and direction relations between that unit and a stationary unit of matter. 


\section{Intrinsic Nature of Emergence-With Illustrations}

Two units of matter and the distance and direction relations between them constitute a pattern-of-material-organization (Figure 3). When the motion of the one unit changes those extensional relations between the two units, a different pattern-of-organization comes into existence that was not there before - a new pattern-of-material-organization emerges (Figures 4 and 5).

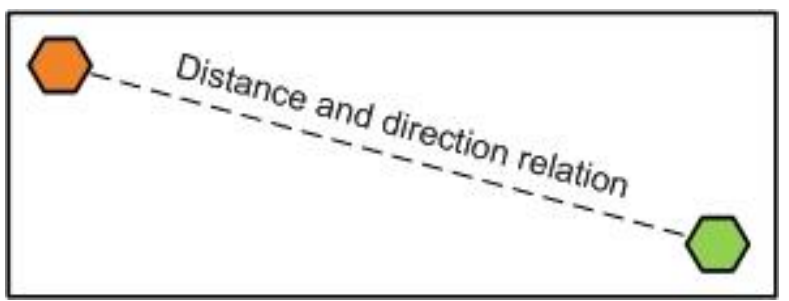

Figure 3. Two units of matter and the distance and direction relations between them constitute a pattern-of-material-organization.

Emergence is intrinsically determinate in that, in the process of emergence, the existence and intrinsic qualities of what goes before determine the existence and intrinsic qualities of what follows. The locations of two units of matter determine the distance and direction relations between them. The qualities of an undisturbed motion at one moment, its speed and direction, determine those qualities of the motion in the following moment (Figure 6 ). The location, speed, and direction of a moving unit at one moment determine the following location of that unit. The new location of the moving unit determines new distance and direction relations between the moving unit and a stationary unit. The location of the stationary unit, the new location of the moving unit, and the distance and direction relations determined by those locations determine a new emergent pattern-oforganization.

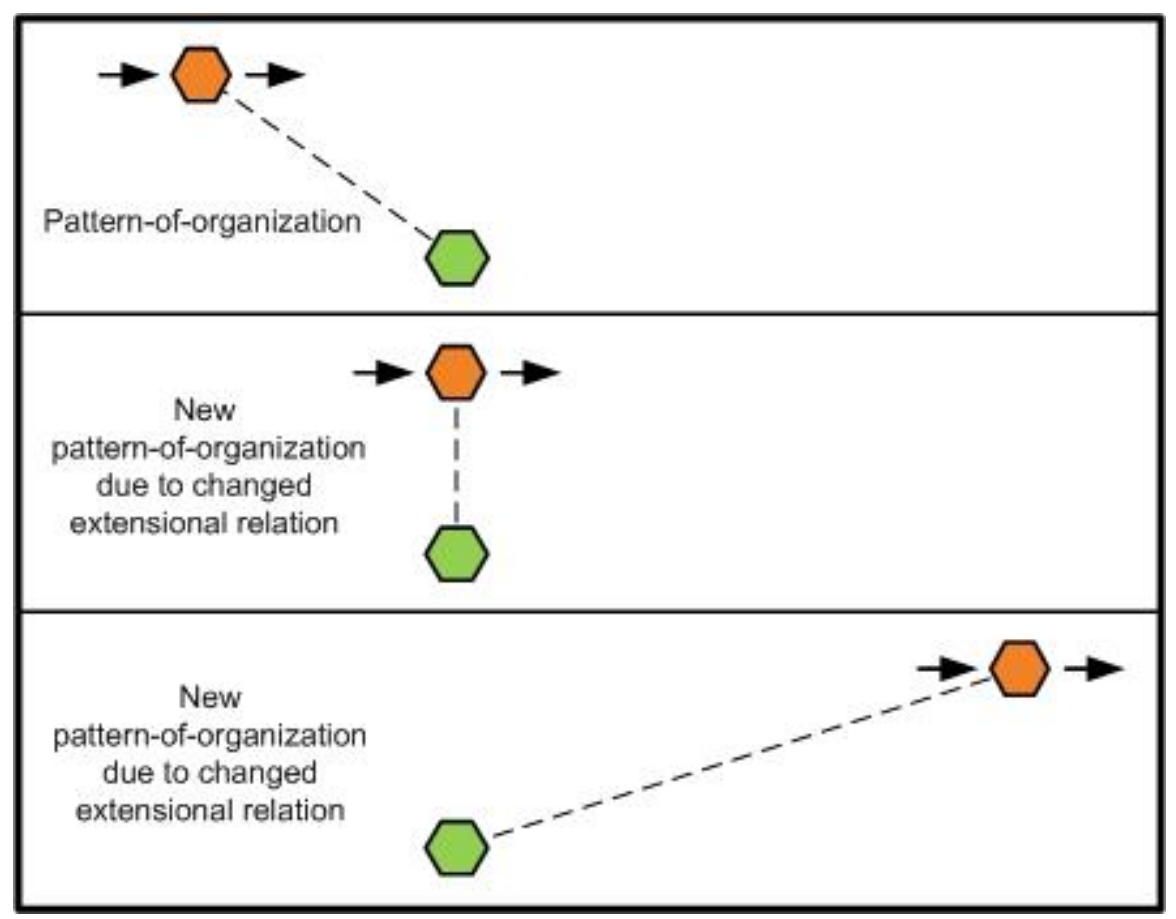


Figure 4. New pattern-of-organization emerges when motion changes the extensional relations between two units.

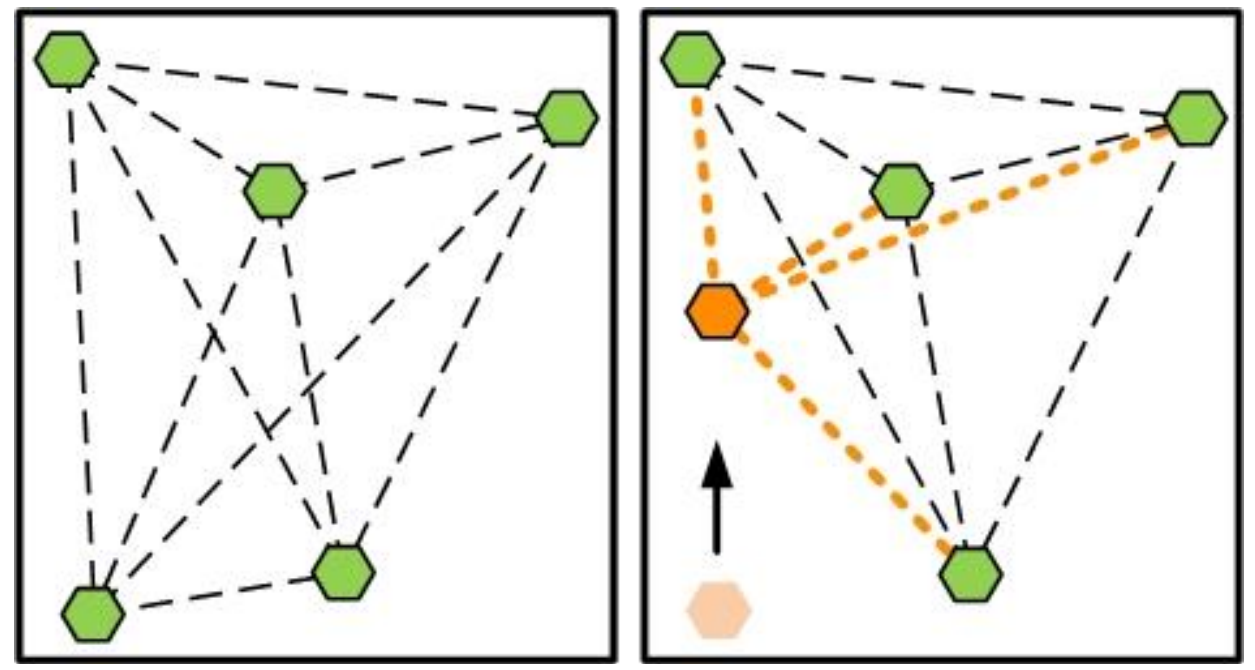

Figure 5. When motion changes any distance or direction relation, new pattern comes into existence - new pattern emerges.

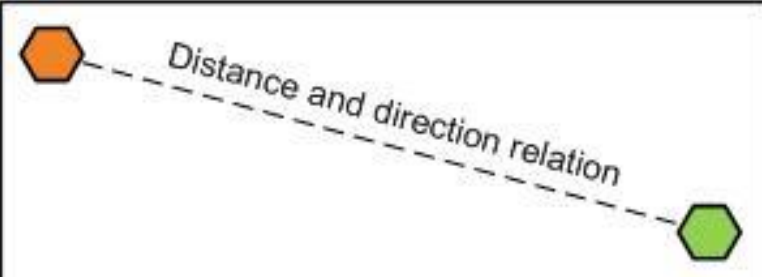

Locations determine relations

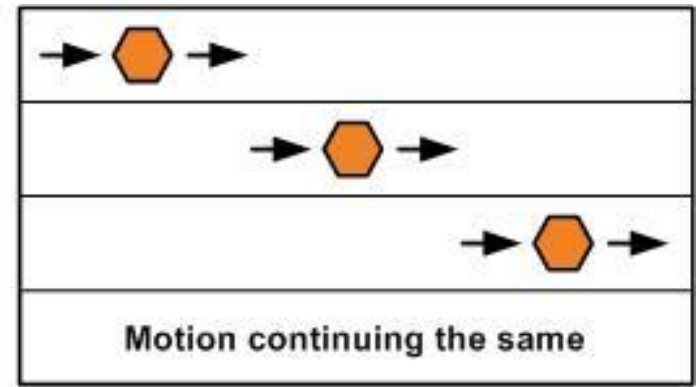

Figure 6. The locations of units of matter determine their extensional relations, and the qualities of a motion at one moment determine the qualities of that motion in the following moment.

Because the motion is ongoing, because it is continuous, the distance and direction relations are changing in a continuous manner. As these extensional relations between the units continuously change, there is continuous change in the pattern-of-organization of the two units - continuous emergence of newly occurring pattern-of-organization.

Emergence is a form of development. Development is a transition, a sequentialdifference, with some form of enhancement. At the development-of-origin of emergence, motion initiates changes in the distance and direction relations between two units of matter, with consequent changes in the pattern-of-organization of the units, and the emergence of new pattern-of-organization. Motion is an intrinsically sequential process, and the consequent changes in extensional relations, pattern-of-organization, and emergent pattern are thereby equally sequential. There is a sequential-difference from one part of an ongoing motion to the following part. There is likewise a sequential-difference 


\section{Intrinsic Nature of Emergence-With Illustrations}

from one distance relation to the next distance relation, from one direction relation to the next direction relation, from one pattern-of-organization to the following one.

Development requires enhancement. Sequential-enhancement occurs when there is a sequential increase of quantity. With any form of sequential-difference there is always some form of increased quantity, some form of more. There is more motion continuing on from what has gone before. There are more distance and direction relations following those that have already occurred. And there are more changes in the pattern-oforganization and more emergent patterns-of-organization following those that have already occurred. In each of these cases of sequentially more - of sequential increase of quantity - there is sequential-enhancement. At its foundational-development-of-origin, emergence is a creative process based on sequentiality, on sequential-difference-on sequential-enhancement (Figure 7).

Self-organization, a form of development, occurs when factors intrinsic to a prior stage of development determine organizational factors intrinsic to the following stage. At the development-of-origin of emergence, (a) the two units of matter, (b) the motion, (c) the spatial place in which they exist, and (d) the distance and direction relations between the units are all factors intrinsic to the situation. At this stage of its development, the process of emergence is a process of self-organization.

\section{Sequential-Enhancement-Emergence}

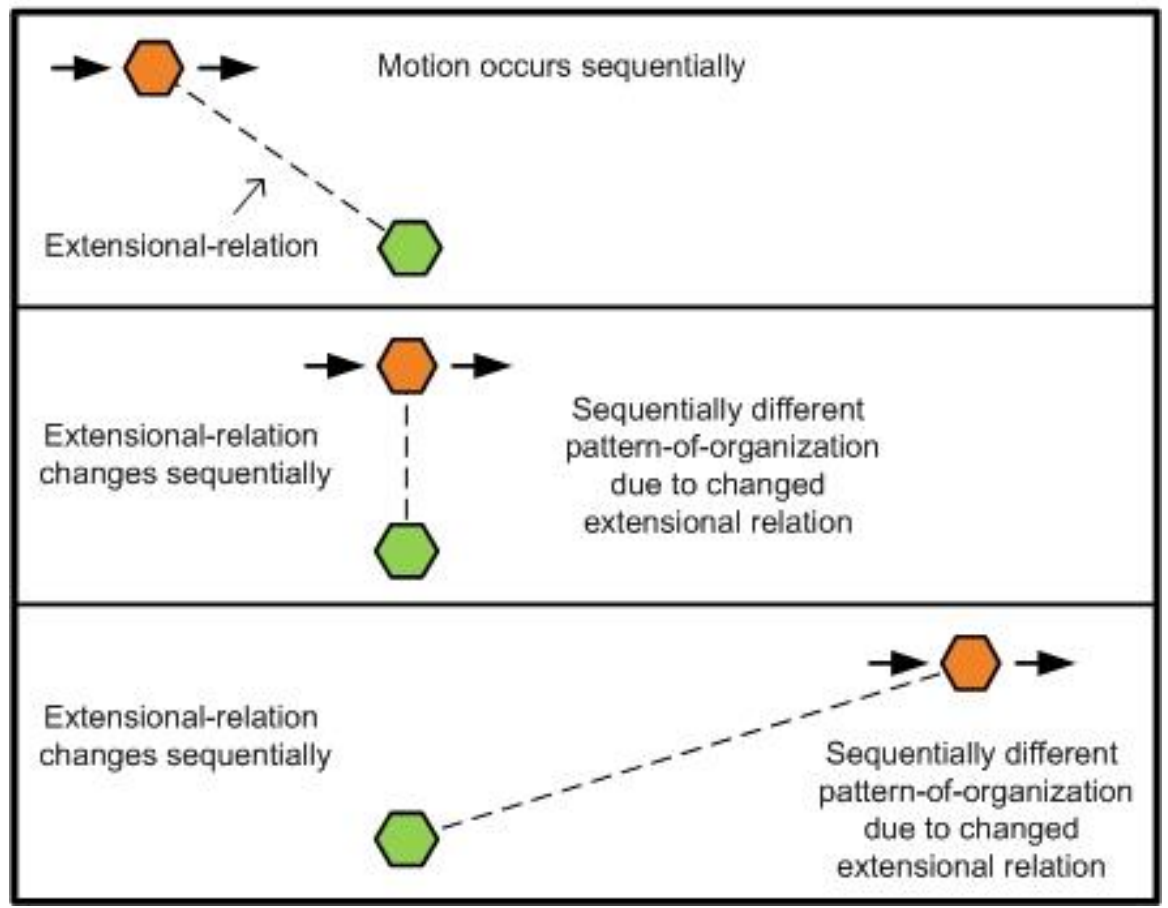

Figure 7. Emergence is based on sequentiality - sequentiality of motion, of newly occurring extensional relations, of newly occurring pattern-of-material-organization. 


\section{Intrinsic Nature of Emergence-With Illustrations}

\section{THE DEVELOPMENT OF EMERGENCE, AND THE ASSOCIATED FACTORS}

Foundationally all forms and cases of emergence are based on (a) material components, (b) relations between those components, (c) pattern-of-material-organization, (d) motion, and (e) sequential-enhancement. There are a great many developed forms of emergence, each one based on additional factors that make it distinct from the other forms. These factors can be used to identify and label the various forms of emergence. The foundational form of emergence, at its development-of-origin, is sequentialenhancement-emergence.

The simplest form of emergence occurs with undisturbed motion of one unit of matter in relation to another unit which is not moving. There are three basic configurations of the motion and the two units. Motion away from the stationary unit. Motion passing by the stationary unit. And motion directly at the stationary unit. Each of these configurations has different consequences for the development of emergence. With motion away there is no development of emergence. With motion passing by there are two major developments of emergence, one involving only one level of the hierarchic organization of material-reality, and the other involving the transition from one level to another. Motion directly at the stationary unit results in a series of major developments in the nature of emergence.

\section{Motion Away from the Stationary Unit}

With motion away, the emerging patterns-of-organization between the units are different from one another by way of the continuously increasing distance factor. No additional factors play roles in the situation, there is no change in the process of emergence, and therefore no factor-development for emergence.

\section{Motion Passing by the Stationary Unit}

With this configuration of the motion in relation to the locations of the units, there are developments-of-origin of two different forms of emergence.

\section{Emergence Due to Transformation-Point}

Except for motion away from or directly toward the stationary unit, all pathways of the moving unit will take it past the stationary unit. This passing-by motion will initially bring the moving unit progressively closer to the stationary unit, then, past the point of closest approach, the motion will take the moving unit progressively further away. At the point of closest approach, a change occurs in the process of emergence. At first, as the moving unit approaches the other unit, the process of emergence of new pattern is in large part based on the decreasing distance between them. Then, past the closest point, the process of emergence is in equally large part based on increasing distance between the units. The motion in association with the point of closest approach initiates a transformation in the basis of the process of emergence. The point of closest approach plays the role of a transformation-point (Figure 8).

A transformation-point is a location or stage in the existential-pathway-development of a situation at which abrupt change occurs that is due solely to the ongoing changing relations between the intrinsic qualities of the components of the situation. The role of a 


\section{Intrinsic Nature of Emergence-With Illustrations}

transformation-point (a) occurs at a specific stage in the existential-pathway-development of the situation, (b) is of short duration, and (c) does not continue on in the existentialpathway-development of the situation. While the role of initiating change is of short duration, the initiated change does continue on. With the ongoing motion, the process of emergence continues - in the newly initiated form. The role of the transformation-point in changing the basis of emergence is determinate in that what goes before determines what follows.

The initiated change in the process of emergence is a change in its pattern-oforganization - from emergence based on decreasing distance relation to emergence based on increasing distance relation. The form of the process of emergence past the point of closest approach, past the transformation-point, is a newly occurring form that was not there before. It is an emergent form of emergence.

\section{Transformation-Point-Emergence}

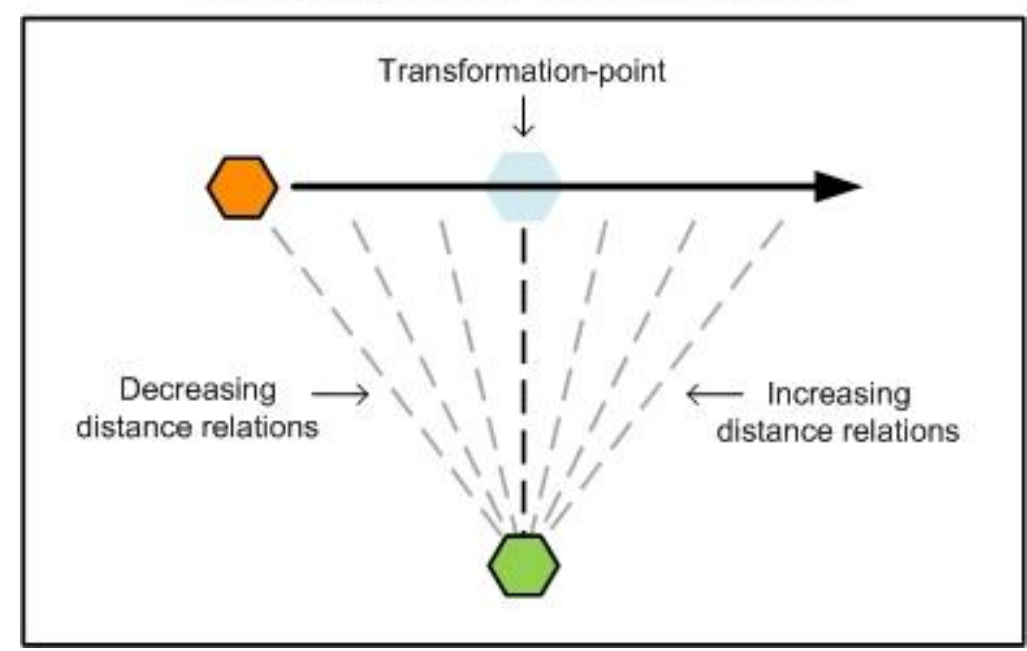

Figure 8. Transformation-Point-Emergence. At the point of closest approach, the basis of the process of emergence is transformed from decreasing distance relation to increasing distance relation.

All developed or higher-level forms of emergence are emergent. An example of particular interest is biological evolution, which is one of the most complex forms of emergence known. Prior to about 3.5 billion years ago there was no life on earth, and therefore no biological evolution. With the origin of life, biological evolution was a newly occurring form of emergence that had not been there before-it was an emergent form.

The change in the process of emergence, initiated by motion in association with a transformation-point, involves development in several ways. It is situation-development due to the combined developments of the motion, the location of the moving unit, and the distance and direction relations. It is existential-pathway-development in that (a) the motion, (b) the prior form of emergence, (c) the passing through the transformation-point, (d) the following motion, and (e) the ongoing new form of emergence constitute the 


\section{Intrinsic Nature of Emergence-With Illustrations}

continuously ongoing sequentially connected development of the situation. It is factor development - direct-transformational-factor-development-because the form of emergence changes, and it does so by the first stage becoming the second stage through the process of the transformation. It is emergence-based development in that a new pattern-of-material-organization comes into existence - a creative form of development. It is self-organizational-development in that, along with the other factors of the process of emergence, the transformation-point is intrinsic to the situation.

The development of emergence initiated by a transformation-point is a type of selforganization. Factor-development occurs as the result of the roles of additional factors. At transformation points factors preexisting in the situation can play new roles, initiating change without any role for a factor extrinsic to the situation. The point of closest approach takes on the role of transformation-point when the moving unit passes through that point, initiating a self-organizational change in the process of emergence.

With passing by motion, the existential-pathway-development of the situation involves a sequence of three forms of emergence, (1) sequential-enhancement-emergence, (2) transformation-point-emergence, and (3) sequential-enhancement-emergence again, but in a modified form. The approach sequential-enhancement-emergence consists of an ongoing series of minor changes. There is no change in the nature of the process of emergence itself. The change from emergence based on decreasing distance to emergence based on increasing distance due to transformation-point-emergence is a major development. This is a change in the nature of the process of emergence. The following, modified, sequential-enhancement-emergence is again an ongoing series of minor changes, without any changes in the nature of the process of emergence.

It often happens that there is an ongoing series of minor changes, then a major change, followed by ongoing minor changes of a different sort. Minor and major developments are typical of existential-pathway-development, and their pattern of interrelation constitutes a general-factor.

A developed form of this pattern of minor and major developments occurs while training personnel in process improvement. (B. Vesterby, personal communication, Apr. 25, 2011) Learning progresses in a series of minor steps, then there is a major step that changes the situation, which is then followed by a now emergently modified series of minor steps. Unexpected major developments can be a source of unintended consequences.

Some factors play roles as the basis of emergence, such as sequential-enhancement in sequential-enhancement-emergence. These factors continue to play roles in the ongoing existential-pathway-development of the situation, roles in maintaining in one way or another the ongoing process of emergence.

Other factors play roles in the initiation of developed forms of emergence, such as the point of closest approach with its role of a transformation-point. As in this case, these factors often do not continue to play roles in the ongoing process of emergence, even 


\section{Intrinsic Nature of Emergence-With Illustrations}

though the consequences of their roles do continue on. In other situations, the factors that play short term roles in initiation continue to play roles in the existential-pathwaydevelopment of the situation, roles in maintaining the changes initiated by their shortterm initiation roles.

The development-of-origin of transformation-point is the beginning of the developments that progress to factors such as threshold and tipping-point, and the emergent forms of emergence initiated by those factors. Factors such as threshold and tipping-point are developed forms of transformation-point. A spillway over a dam is an example of a factor that plays both a change initiating role, as a threshold, and a role in maintaining the newly initiated process.

As the water rises in the reservoir behind the dam, it will eventually reach the level of the spillway, which then plays the transformation-point role of a threshold, allowing the water to flow over the dam. The dam was playing a blocking role to the flow of water down the valley, and thus, in association with the incoming water, was playing a role in the emergence of a lake (Figure 9). When the level of the water reaches the spillway, the process of creating the lake ends and the process of water flowing down the valley reemerges. The spillway plays the role of a threshold that changes the processes of water flow from that of creating a lake to that of ongoing river flow. Because the spillway is the channel for the new flow, it is also playing a role in maintaining the characteristics of that flow. It plays a role in maintaining the emergent form of moving water.

The spillway, as a threshold-transformation-point has a role that initiates change. It changes the configuration of the flow. The role in the initiation (a) occurs at a specific stage in the existential-pathway-development of the situation, (b) is of short duration, and (c) does not continue on in the existential-pathway-development of the situation. While the role of the spillway in changing the configuration of the flow of water is of short duration, the consequent change in configuration continues on. The spillway, as a channel through which water flows, has a role that maintains the initiated change. It maintains the configuration of the emergent flow, and in doing so this role continues, in contrast to the initiation role (Figure 10). 


\section{Intrinsic Nature of Emergence-With Illustrations}

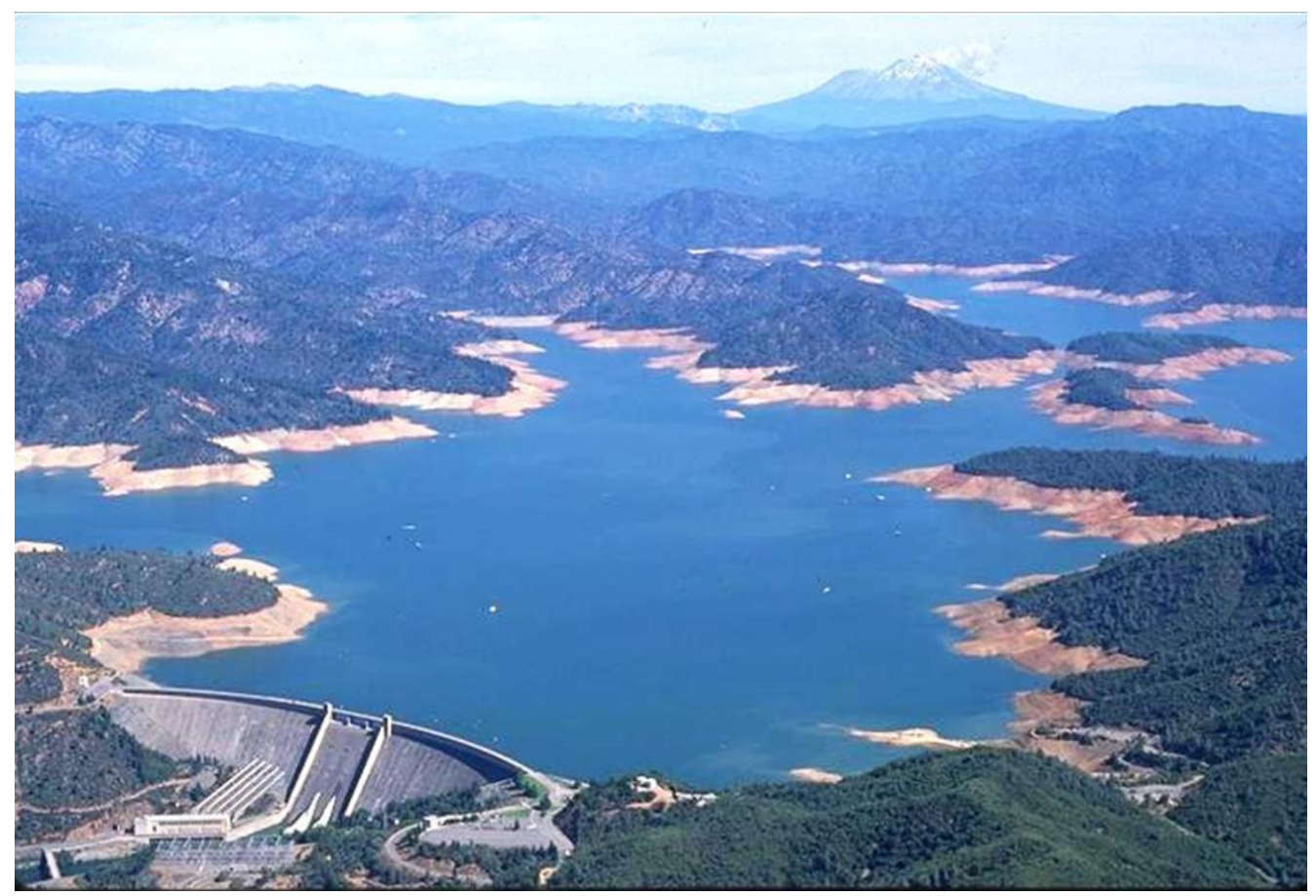

Figure 9. A dam playing a blocking role to the flow of water, and thereby playing a role in the emergence of a lake. (Courtesy U.S. Bureau of Reclamation.)

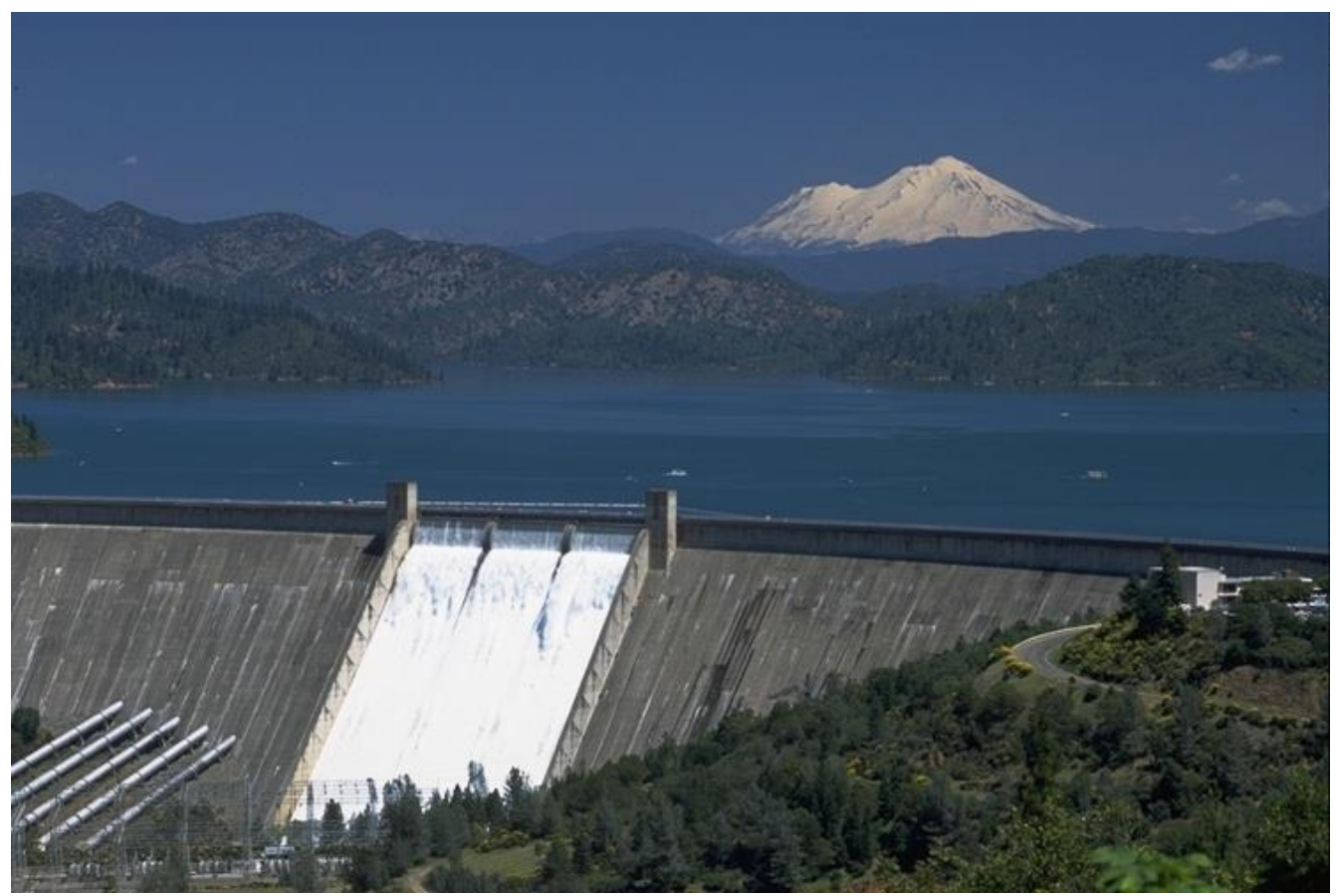

Figure 10. Threshold-Transformation-Point. At the spillway threshold, the lake filling process is transformed into a river flow process. (Courtesy of Redding Chamber of Commerce.) 


\section{Intrinsic Nature of Emergence-With Illustrations}

Because transformation-points and their developed forms are factors intrinsic to the situations in which they occur, sometimes without any outside signs of their presence, they can be hard to anticipate. Thresholds, tipping-points, and other forms of transformation-points can be a source of unexpected consequences.

\section{Emergence Due to Combinatorial-Enhancement}

Motion changing the location of one unit in relation to the location of another unit constitutes a rearrangement of the components of their pattern-of-organization, a reorganization of that pattern. Hierarchically, this is occurring at one level, at one stage of the hierarchic-organization of material-reality, a horizontal reorganization. When motion in relation to a transformation-point changes the basis of an ongoing process of emergence, from decreasing distance to increasing distance, that constitutes a reorganization of the manner in which the extensional relations are changing. This also is a horizontal or one level reorganization. At these stages of its development, at this level, sequential-enhancement-emergence is a creative process of rearrangement, a process of reorganization of the types of relations that are already factors of the situation.

In a situation where the two units are initially a long way away from one another, but in which the motion of the one unit is toward the stationary unit, the motion brings the units progressively closer together. Sequential-enhancement-emergence occurs continuously as the distance between the units decreases. When the units are close enough, a different type of relation occurs between the units, resulting in a developed form of enhancement and a developed form of emergence. At this stage of its development, emergence becomes a creative process based on coming together, on combining-on combinatorialenhancement.

Just as sequential-enhancement-emergence develops directly into transformation-pointemergence when the existential-pathway-development of the situation reaches the appropriate stage, sequential-enhancement-emergence can also develop directly into combinatorial-enhancement-emergence. Combinatorial-enhancement-emergence is a process of rearrangement, a process of reorganization of the types of relations that are already factors of the situation - that results in the origin of a newly occurring type of relation. When units come together a relationship develops that was not there before, the group relation - a group emerges. This is a development-of-origin of the general-factor group. While motion and sequentiality play roles in bringing the units together, it is the combining, the togetherness, the proximity of the units that result in the new form of enhancement. The enhancement in this situation - the more - is the group relation, the emergence of the group, and the emergence of hierarchic organization (Figure 11). 


\section{Intrinsic Nature of Emergence-With Illustrations}

\section{Combinatorial-Enhancement-Emergence}

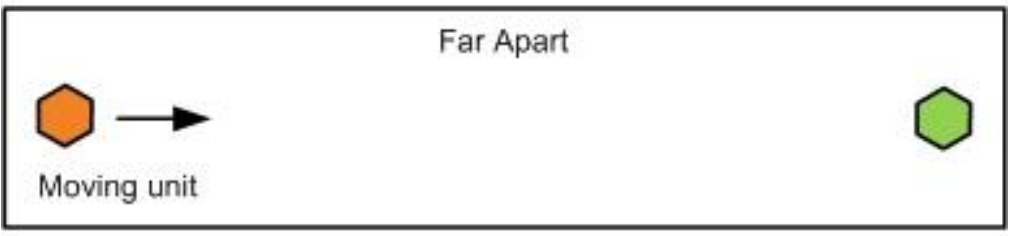

In Proximity

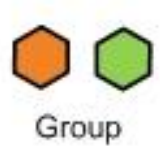

Figure 11. Combinatorial-Enhancement-Emergence. When motion brings units of matter into proximity, the group relation emerges.

With passing by motion, with no other factors playing roles, the motion continues on, and the unit passes the point of closest approach and moves away. The stationary unit and the region of group relation are left behind. While the group relation lasts longer than the transformation-point relation, occurring in a region of space rather than a point in space, with motion passing by, the group relation is still a short-term occurrence.

A group is not just a distance and direction-based pattern-of-material-organization, such as the emergent patterns that result from sequential-enhancement-emergence, when the units are further apart. A group is a specific type of pattern-of-organization that can play roles that do not occur when the pattern of the units is more spread out. It is a group when the units play roles together that cannot be played by the individual units when they are not together.

A group can play a role as a factor in the situation in which it occurs. Probably the simplest form in which a group occurs in relation to a larger context is when the proximity of the units in the group is greater that their proximity to other surrounding units. In another situation, two units constitute a group when they are close enough together such that another unit cannot pass between them without colliding with one or the other or both. Roles of both units are required simultaneously to impede the free passage of the third unit.

A developed, higher level, example of (a) units combining, (b) the consequent emergence of a group, and (c) the occurrence of group level roles, occurs when a gull resting on a beach is joined by another gull. A group of two gulls has emerged. As time goes by, other gulls come along, see the group, and join it. The group can grow to dozens or even hundreds of gulls, terns, sandpipers, pelicans, and plovers, resting, preening, or sleeping all together.

When a Bald Eagle, large falcon, or a dog comes near the group, gulls which are not sleeping give alarm calls, warning the flock and awakening the sleeping birds, and the entire group takes flight. Group level roles occur, both internal to the group (the alarm 


\section{Intrinsic Nature of Emergence-With Illustrations}

calls communicating the threat to all the units of the group), and in relation to the external context, (the response of the entire flock to the approach of a predator).

While the locations and qualities of transformation-points in an existential-pathwaydevelopment are generally specific, the origins and intrinsic qualities of groups in the general-development-of-reality are not always so precise. For example, how close two units must be to play a group role that obstructs the free passage of another unit depends on the size of that third unit (Figure 12). Nonetheless, a group is a general-factor, an intrinsic quality of the general-development-of-reality, with proximity of the units establishing the distinction between the group and surrounding units.

Group Relation

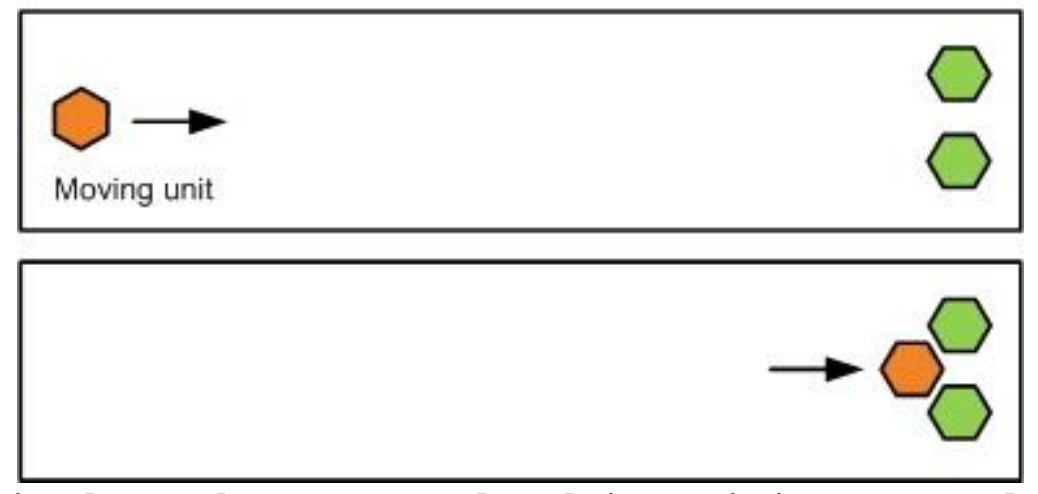

Figure 10. Units play a role as a group when their proximity prevents the free passage of another unit.

At developed stages, the moment of origin of a group can be more specific, as when two atoms join to form a molecule. In the case of the gulls, the group grows as additional birds land by the others on the beach. The group originates in the same way. When the second gull flew up to the first bird that was already on the ground, the two of them formed a group in a simple manner as in the case when a moving unit came close enough to a stationary unit to form a group. And as in that prior situation, if the flying gull ignores the landed gull and passes on by, the group relation is left behind. However, if the flying gull lands near the other gull, the group relation is stabilized. A social relation is established between the birds, and a social group emerges. This is a specific development-of-origin of the group.

The development-of-origin of group is concurrent with the development of origin of hierarchy. While the sequential-enhancement-emergence leading up to combinatorialenhancement-emergence is a one level process, combinatorial-enhancement-emergence is a bilevel process in that it creates the next level, the next stage of hierarchic-organization. A group is a whole composed of units. This is hierarchic organization, with the group as a whole forming the upper level, (with group level roles), and the units forming the lower level, (with lower level roles, such as constituting the deep-structure of the upper level). 


\section{Intrinsic Nature of Emergence-With Illustrations}

The origin of a group is based on the coming together of the component units of the group - a combining of units. Sequentiality alone does not necessarily result in combining. There must be the additional factor that the motion is in a direction toward the stationary unit. Direction plays a required role. The direction must bring the moving unit close enough to the stationary unit such that a group relationship emerges, such as when the units play roles together that cannot be played by the individual units when they are not together.

\section{Motion Directly at the Stationary Unit}

Motion directly at the stationary unit results in a series of major developments - the emergence of (1) collision, (2) contact, (3) push (cause), (4) throughflow, and (5) coherence between units of matter. The existential-pathway-development that leads up to collision requires a specific role for direction - it must be directly at the stationary unit such that the moving unit cannot pass by without hitting the stationary unit. There is continuous sequential-enhancement-emergence as the moving unit approaches. Because there is no passing by, there is no passing by point of closest approach, and no passing by type of transformation-point. When the moving unit is close enough, the group relation emerges, and because the moving unit does not pass by, the emergent group remains a factor through the following existential-pathway-developments. The emergence of collision is due to the combined roles of sequential-enhancement-emergence, combinatorial-enhancement-emergence, and the required role of the appropriate direction of motion. The collision event itself is complex, involving an existential-pathwaydevelopment with (a) major developments of emergence and (b) developments-of-origin for other factors. A collision is a developed form of transformation-point with abrupt changes in various factors of the situation as a consequence of the ongoing existentialpathway-development of the situation

\section{Emergence due to substantiality-based-combinatorial-enhancement.}

The stationary unit is in the way of the moving unit, blocking its path. When the moving unit collides with the stationary unit, a factor already present takes on an additional role. That factor is material substantiality.

The substantiality of matter is different from the immateriality of space-something isthere that can play roles that are impossible for immateriality. Some of these roles occur in the foundational general-development-of-reality prior to the development-of-origin of emergence. Substantial matter occupies immaterial spatial-place. Units of matter play roles in patterns-of-material-organization. And it is matter that moves. Other roles of substantiality are consequences of emergence, the first of which occurs in the collision situation.

When the units collide, substantiality plays a role in combinatorial-enhancement, which results in a new form of emergence-adjacent-substantiality-based-emergence. The developmental-enhancement - the more that now occurs in the situation - is the emergent factor contact between the substantiality of the one unit and the substantiality of the other unit. 


\section{Intrinsic Nature of Emergence-With Illustrations}

Contact is a supraorganizational-factor, a factor that is more than organizational in nature. All prior emergent factors were patterns-of-material-organization, and organizational factors do play roles in the development-of-origin of the supraorganizational-factor, and in the organization of the situation in which it occurs. It is a group pattern-of-material-organization in which the units have arrived in an adjacent relation wherein the additional role of substantiality results in contact. The role of substantiality is required for the emergence and ongoing existence of supraorganizational factors.

\section{Emergence due to blocked motion and contact-based-combinatorial-enhancement}

The stationary unit blocks the motion of the other unit, and the blocked motion results in a push against the stationary unit. The push is the developmental-enhancement at this stage of the existential-pathway-development of the collision event. This is blockedmotion-based-emergence, and the push is the emergent factor.

In the collision of a moving unit of matter with a stationary unit, adjacent-substantialitybased-emergence results in contact between the units. In this origin of contact, it is the mere existence together, in adjacent relation, of the substantiality of one unit with the substantiality of the other unit that, through substantiality-based-combinatorialenhancement, results in the emergence of this supraorganizational-factor.

Contact, as an additional factor in the situation, then plays a role connecting the moving substantiality role of one unit with the blocking substantiality role of the other unit, with the consequent emergence of another supraorganizational-factor-push - the core factor of cause. In the origin of push, it is not just the existence together of two units of substantiality that results in emergence, but rather the existence together of the roles of the two units of substantiality that, through contact-based-combinatorial-enhancement, results in the interrelation of these roles and the emergence of this second supraorganizational-factor, push.

\section{Emergence due to push}

With contact, the substantiality of the stationary unit plays the emergent role of blocking the motion of the other unit. When blocked, the substantiality of the moving unit plays the emergent role of pushing against the substantiality of the stationary unit. The blocked forward motion of the moving substantiality is transformed into a pressure wave that propagates through the substantiality of the stationary unit.

At this stage of the situation-development, push is the additional factor that now plays a role in the emergence of cause-based-emergence. The pressure wave is the developmental-enhancement, the emergent factor. The push from one unit to the other unit causes the emergence of the pressure wave.

When the substantiality of the moving unit presses against that of the stationary unit, transforming the motion of the one unit into a pressure wave through the second unit, the units and roles are already combined by way of contact. The transformation of the motion to a pressure wave is sequential through time, space, and matter-from the substantiality 


\section{Intrinsic Nature of Emergence-With Illustrations}

of one unit to the substantiality of the other unit. Cause-based-emergence is due to sequential-enhancement rather than combinatorial-enhancement, as in the previous two stages of the development of emergence.

When the motion is blocked, that motion gets transformed from that of a unit of matter passing through space to the motion involved in the propagation of the pressure wave through the blocking unit. The internal matter of the unit gets shifted about as the push involved with the propagation of the wave moves through the matter of the unit. Blocking matter alters the flow of motion, and the flow of motion alters blocking matter. In other words, blocking matter alters the flow of energy, and the flow of energy alters the organization of blocking matter. This relation is a major source of situation development, existential-pathway-development, and the development of complexity. It is also a core factor in many developed forms of emergence.

The pressure wave, by way of existential-pathway-development as it passes from one side of the stationary unit to the other side, becomes a development-of-origin for throughflow. Throughflow is the passage of push, matter, or pattern-of-organization, or some combination thereof, through a situation or system.

The pressure wave is a form push, and in this foundational form of throughflow, it is this push that is the primary factor that progresses through the unit from one side to the other. The push creates the pressure wave pattern-of-organization, which passes through the unit along with the push. The matter of the stationary unit gets shifted as the pressure wave occurs, and the matter of the unit thus plays a role in the propagation of the push and the pressure wave pattern, but no matter passes all the way through the unit with the push and the wave pattern.

\section{Emergence due to throughflow}

The descriptive definition of throughflow includes push, matter, and pattern-oforganization as factors that flow through situations and systems. The roles of these factors vary greatly from one form of throughflow to another. With this foundational form, which occurs in collision situations, it is push and the pattern of a pressure wave that pass from one side of the unit to the other side. With a river flowing down a valley, it is matter that has the primary role for throughflow, albeit carrying with it a considerable push of moving water. Pattern-of-organization plays a lesser role as in the direction of flow downstream, and in the patterns within the turbulence carried along with the flowing water.

In the prior forms of emergence, it has been the case that one additional factor resulted in a development of the nature of emergence - factors such as (a) transformation-point, (b) combinatorial-enhancement, (c) substantiality, (d) contact, and (e) push. Sometimes the additional factor was a new role for a factor already present in the situation, such as point of closest approach and substantiality. Other times the additional factor was an emergent factor, such as contact and push. Emergence due to throughflow is more complex. 


\section{Intrinsic Nature of Emergence-With Illustrations}

This form of emergence is based both on the interrelations of the factors that take part in the throughflow - push, matter, and pattern-of-organization - and on the interrelations of the flow of these three with the situations and systems through which they flow. The pressure wave momentarily shifts the matter of the unit - momentarily reorganizing the matter, and creating a short-term emergent pattern-of-organization. In a river, the downstream push of the flowing water and the downstream movement of the pattern of turbulence shift the sand, gravel, and stones in the riverbed, pounding them against the substrate and wearing it away. The channel is progressively reshaped, and the river takes on a new emergent pattern-of-organization as it wears its way deeper into the substrate and shifts the channel back and forth over geologic time from one side of the valley to the other.

When playing a role in the process of emergence, throughflow reorganizes the situation or system through which it flows. New pattern-of-organization emerges. This can take place through a single pulse, as with the collision pressure wave, or by way of a continuous throughflow, as in the case of the river. Continuous throughflow can result in the emergence of a steady-state.

\section{A developed stage of emergence due to push}

The initial push of the moving unit on the blocking unit caused a pressure wave to propagate through the matter of the blocking unit. When the pressure wave reaches the far side of the stationary unit, that unit begins to move. The unit as a whole begins to move when the push has reached all parts of the unit. The push from the moving unit causes the blocking unit to move. This motion of the blocking unit was not there beforeit is emergent - a case of caused emergence.

The development-of-origin of the movement of the blocking unit is another situation involving a role for substantiality. While motion involves organizational factors, such as direction of motion, it is matter that moves - substantiality is a required factor in any case of motion. Motion is a supraorganizational factor.

\section{Emergence Due to Coherence}

Units of matter stick together, which constitutes a developed form of combinatorialenhancement. The result is coherence-based-emergence. Group, contact, and hierarchic organization are prior factors that play developed roles in this situation.

Two units that are close together constitute a group, with the simple hierarchic organization of such a group. When the components of the group are in adjacent relation, there is the emergence of the contact relation. Just as contact makes it possible for there to be the push relation, contact makes it possible for there to be bonding between unitsthe coherence relation. At the development-of-origin for group, the relations between the units is loose. They can move freely in relation to one another. Units in coherent relation have much less freedom of motion, and tend to maintain their positional orientation to each other. Structural-hierarchic-organization emerges with the coherent relation. The coherent units have an emergent specific shape as a compound structure, as a whole. 


\section{Intrinsic Nature of Emergence-With Illustrations}

In the next stage of combinatorial-enhancement, the specific shapes of compound structures play roles both in the manner in which these units combine and in the nature of this next stage of emergent shape of compound structure. In situations where there are few units in the compound structure and the sizes of these units are large relative to the size of the emergent whole, the shapes and other qualities of the individual units play dominate roles in the emergent qualities of the emergent whole. In situations composed of ever larger numbers of coherent units, the roles of the individual units become progressively less significant in determining the specific qualities of emergent factors of the whole.

In small molecules the qualities of the component atoms determine the shape of the molecule. In large molecules, such as a protein, the individual molecules each still play significant roles, but it is a developed form of emergence, involving the folding of the polypeptide strand of which the protein is made, that determines the overall shape of the emergent protein. In a material such as wood, the atoms and molecules play very small roles in the shape a sculptor can give to an emergent work of art.

\section{CONCLUDING REMARKS}

Emergence is a consequence of the interrelations between factors. The new existence, and the intrinsic qualities, of an emergent factor are the consequences of what factors are playing roles, the intrinsic qualities of those factors, and the manner of togetherness of those factors. There are certainly tens of thousands of different forms of emergence, each one based on a unique group of components, qualities, and manner of togetherness.

The key to understanding this diversity of creative processes is to understand the generalfactor development. Everything that exists takes part in one way or another in a universally omnipresent transition, a sequential-difference from one time, place, part, pattern, level, condition, or situation to another involving some form of enhancement. Development is universal, and can thereby be used as a unifying principle for all knowledge of the intrinsic nature of reality.

There are intrinsic qualities of development that provide conceptual access to its unifying universality. First, there are many different kinds of development, each one providing a specific conceptual tool for understanding and sequentially unifying some developmental aspect of reality. Achieving an understanding of each type, and then combining them in the manner of their natural interrelations in the general-development-of-reality provides broad and deep insight into the development and diversity of emergence. Second, all the different types of development have developmental directionality. This sequential directionality can be used to provide conceptual orientation, which in turn integrates in parallel all the forms of development that are taking place in the existential-pathwaydevelopment of any particular situation. Understanding developmental directionality provides orientation within the general-development-of-reality. Emergence is a primary factor of creative directionality. And third, the various types of development each occur by way of a series of stages. Understanding stages provides conceptual location in particular developmental sequences, such as that of the general-factor, emergence. 


\section{Intrinsic Nature of Emergence-With Illustrations}

Understanding stages additionally provides conceptual location in the generaldevelopment-of-reality.

The basic form of any general-factor is present within all developed forms of that general-factor, giving the developed forms their identity as that general-factor. The basic form of emergence is present in all its developed forms. Any prior form can exist within and play roles that make more developed forms what they are. All developed forms of any general-factor, and all situations of whatever complexity, are composed of many general-factors in various stages of their developments. Highly developed processes of emergence, such as biological evolution, ontogeny, and cultural development, have many developmentally prior forms of emergence playing roles within them, making it possible for the higher forms to exist.

Further explanation of emergence can be found at:

Vesterby, Vincent. 2019. "Emergence Is an Isomorphy." OSF Preprints. July 4. doi:10.31219/osf.io/ys3wd.

Vesterby, Vincent. 2019. "Emergence Is Why It Is Not Possible to Explain Life Solely with Physics and Chemistry.” OSF Preprints. July 9. osf.io/cwnt7.

\section{REFERENCES}

Bertalanffy, L. von. (1968) General System Theory. George Braziller, New York.

Lewes, G. H. (1891) Problems of Life and Mind. First Series, The Foundations of a Creed. Vol. 2., Houghton, Mifflin and Co., Boston.

Mill, J. S. (1843) A System of Logic, (Eight edition, 1872)., Longmans, Green and Co., London.

Vesterby, V. (2008a) Are Ecosystems Alive? Proceedings of the 52 ${ }^{\text {nd }}$ Annual Meeting of the ISSS, Madison, Wisconsin, USA. http://journals.isss.org/. Accessed 15 May 2011.

Vesterby, V. (2008b) Origins of Self-Organization, Emergence and Cause. ISCE Publishing, Goodyear, AZ.

Date of original version of “The Intrinsic Nature of Emergence”-July 2011. 